\title{
Burden of community-onset bloodstream infections, Western Interior, British Columbia, Canada
}

\author{
K. B. LAUPLAND ${ }^{1,2} *$ K. PASQUILL ${ }^{3}$, E. C. PARFITT ${ }^{1}$, P. NAIDU $^{4,5}$ AND \\ L. STEELE ${ }^{3}$ \\ ${ }^{1}$ Department of Medicine, Royal Inland Hospital, Kamloops, British Columbia, Canada \\ ${ }^{2}$ University of Calgary, Calgary Alberta, Canada \\ ${ }^{3}$ Department of Pathology and Laboratory Medicine, Royal Inland Hospital, Kamloops, British Columbia, \\ Canada \\ ${ }^{4}$ University of Alberta, Edmonton, Alberta, Canada \\ ${ }^{5}$ Provincial Laboratory for Public Health, Edmonton, Alberta, Canada
}

Received 9 January 2016; Final revision 13 February 2016; Accepted 2 March 2016; first published online 21 March 2016

\section{SUMMARY}

Although community-onset bloodstream infection (BSI) is recognized as a major cause of morbidity and mortality, its epidemiology has not been well defined in non-selected populations. We conducted population-based surveillance in the Interior Health West region of British Columbia, Canada in order to determine the burden associated with community-onset BSI. A total of 1088 episodes were identified for an overall annual incidence of 117.8/100 000 of which 639 (58.7\%) were healthcare-associated (HA) and $449(41 \cdot 3 \%)$ were community-associated (CA) BSIs for incidences of $69 \cdot 2$ and 48.6/100 000, respectively. The incidence of community-onset BSI varied by age and gender and elderly males were at the highest risk. Overall $964(88.6 \%)$ episodes resulted in hospital admission for a median length of stay of 8 days; the total days of acute hospitalization associated with community-onset BSI was 13530 days or 1465 days/100 000 population per year. The in-hospital mortality rate was $10 \cdot 6 \%(102 / 964)$ and this was higher for HA-BSI $(72 / 569,12 \cdot 7 \%)$ compared to CA-BSI $(30 / 395,7 \cdot 6 \%, P=0 \cdot 014)$ episodes. Community-onset BSI, especially HA-BSI, is associated with a major burden of illness.

Key words: Bacterial infections, bloodstream infections, epidemiology.

\section{INTRODUCTION}

Community-onset bloodstream infections (BSIs) are those that are identified in outpatients or first cultured within $48 \mathrm{~h}$ of admission to hospital $[1,2]$. These may be further classified as healthcare associated (HA) or community associated (CA) when related to significant prior healthcare exposure or not, respectively [3]. Although community-onset BSIs are common, their overall epidemiology has not been well defined.

\footnotetext{
* Address for correspondence: Dr K. B. Laupland, Royal Inland Hospital, 311 Columbia Street, Kamloops, BC, V2C 2T1, Canada. (Email: klaupland@gmail.com)
}

Previous studies have largely been hospital-based series that are subject to selection bias and lack denominators for establishing incidence rates or have been focused on selected aetiologies or patient subgroups [4-15]. Only a few population-based studies conducted in Canada, Denmark, and the United States have been reported that have investigated all communityonset BSIs occurring at the population level during the past three decades [16-20]. Only one of these has reported both incidence and mortality outcome for $\mathrm{HA}$ and CA infections [18].

It is important to establish the burden of communityonset BSIs in order to prioritize healthcare funding and research efforts. Therefore, the objective of this 
study was to conduct population-based surveillance in the Interior Health West (IHW) region of British Columbia, Canada in order to establish the burden of HA and CA community-onset BSIs.

\section{METHODS}

\section{Study population}

Interior Health is one of five health regions within the province of British Columbia, Canada. It administers virtually all healthcare to the residents of the southern interior of British Columbia [21]. The IHW region (2012 population: 184010 ) of Interior Health includes the city and surrounding population of Kamloops (2012 population: 110256) and a number of other communities distributed over a large and geographically diverse area. Within Interior Health, patients may be admitted to one of seven hospitals of which tertiary care is provided at Royal Inland Hospital in Kamloops [22]. Only patients requiring acute bone marrow or organ transplantation, major burns, cardiothoracic surgery and a few other highly specialized services are routinely referred elsewhere either within the region or in the province. All residents of Interior Health as identified by postal code with community-onset BSIs during the 5-year period between 1 April 2010 and 31 March 2015 were included in this study.

\section{Ethics statement}

The regional research ethics review board approved this study. The authors assert that all procedures contributing to this work comply with the ethical standards of the relevant national and institutional committees on human experimentation and with the Helsinki Declaration of 1975, as revised in 2008

\section{Population-based surveillance}

An active, population-based surveillance design was utilized. All BSIs occurring in Interior Health residents were identified through the regional hospital laboratory at Royal Inland Hospital. This laboratory performs microbiology testing on all samples submitted from all hospitals, other institutions, and the community within the IHW region. Demographic, clinical, hospital length of stay, and in-hospital mortality outcome information was obtained by individual case review of files from the regional clinical information system.

\section{Laboratory procedures and definitions}

All blood was cultured using the BacT/Alert 3D System (bioMérieux, France). A blood culture set consisted of an aerobic/anaerobic bottle pair obtained from a single draw. Standard practice during this study period was to draw two sets of blood cultures from different sites. Organisms were isolated and speciated using standard methods. A BSI was defined as the growth of a pathogenic organism from at least one set of blood cultures. Organisms frequently associated with contamination including coagulase-negative staphylococci, viridans group streptococci, or Bacillus, Corynebacterium, or Propionibacterium species were $a$ priori required to have at least two sets of blood cultures positive within a 5 -day period to be considered for inclusion in analysis [23]. These were further confirmed by an individual review of detailed microbiology, radiological, and clinical information. Repeat isolations with the same species in a given patient within 30 days were considered to represent the same episode and only those repeat isolations within 30 days to 1 year were classified as new incident infections if the initial episode was treated with clinical resolution. Community-onset BSIs were classified as those obtained from patients that were not admitted to hospital or identified within the first $48 \mathrm{~h}$ of admission to an acute-care hospital [24]. These were further classified as either HA or CA using the definitions of Friedman et al. [3]. Long-term-care facility or nursing-home residents, patients who had specialized care including intravenous therapy at home or who attended a hospital or haemodialysis clinic within 30 days prior to BSI, or those admitted for $>2$ days in the preceding 90 days were deemed to have HA infections and if none of these criteria were fulfilled then they were classified as having CA BSIs. Polymicrobial BSIs were those that had more than one species co-isolated within an episode of disease.

\section{Statistical analysis}

All analyses were performed using Stata v. 12 (StataCorp, USA). Differences in proportions in categorical data were assessed using Fisher's exact test. Medians with interquartile range (IQR) were used to describe skewed continuously distributed variables. Incidence rates were calculated using regional census data [25]. Age- and gender-specific risks were calculated and reported as incidence rate ratios (IRRs) with $95 \%$ confidence intervals (CIs) as described previously [26]. 


\section{RESULTS}

During the 5 years of surveillance, a total of 1088 episodes of community-onset BSIs were identified in 996 IHW residents for an overall annual incidence of $117 \cdot 8 / 100000$ population. Six hundred and thirty-nine $(58.7 \%)$ were HA and $449(41 \cdot 3 \%)$ were CA for incidences of $69 \cdot 2$ and $48 \cdot 6 / 100000$, respectively. Seventy-one $(7 \%)$ patients had second incident episodes of BSI and $16(2 \%)$ had three, four had four, and one patient had five episodes during the course of the study. While the annual incidence rates were similar during 2010-2013 (Fig. 1), there was a significant increase observed in 2014 compared to the previous study years $(154 \cdot 5$ vs. $108 \cdot 5 / 100000$, IRR 1·42, 95\% CI $1 \cdot 24-1 \cdot 63, P<0 \cdot 0001)$.

\section{Demographic risk factors}

The median age was 67.9 (IQR, 54.0-78.4) years and $585(53 \cdot 8 \%)$ episodes occurred in males. A relationship was observed between age and gender and the incidence of community-onset BSI for the very young and the elderly at highest risk, as shown in Figure 2. Although the overall incidence of community-onset BSI was higher in males than in females $(125 \cdot 3 \mathrm{vs}$. $110 \cdot 0 / 100000$, IRR $1 \cdot 14,95 \%$ CI $1 \cdot 01-1 \cdot 29, P=$ $0 \cdot 0163$ ), the relationship between gender and risk for community-onset BSI varied significantly by age. While there was no significant difference in risk in those aged $<10$ years (males 36.5 vs. females $28.0 /$ 100000 , IRR $1 \cdot 30,95 \%$ CI $0 \cdot 59-2 \cdot 99, P=0 \cdot 2443$ ), males were at significantly reduced risk in the 10-39 years age group (males $18 \cdot 7$ vs. 39.3/100 000, IRR $0 \cdot 48,95 \%$ CI $0 \cdot 30-0 \cdot 74, P=0 \cdot 0003)$ but at higher risk in those aged $>50$ years (males 255.7-196.8/ 100000 , IRR $1 \cdot 30,95 \%$ CI $1 \cdot 13-1 \cdot 49, P=0 \cdot 0001$ ) with a marked difference in the very old (Fig. 2).

\section{Clinical foci and microbiology}

The urinary tract $(32 \%)$, intra-abdominal/pelvic $(20 \%)$, and respiratory tract $(12 \%)$ were the most common clinical foci of BSI as shown in Table 1. The distribution of clinical foci varied significantly $(P<0 \cdot 001)$ as to whether cases were HA or CA, and this was largely attributable to fewer CA cases being non-focal and a higher proportion being of cardiovascular or central nervous system focus (Table 1).

Although a wide range of organisms caused community-onset BSI, the three species Escherichia coli, Staphylococcus aureus, and Streptococcus pneumoniae were responsible for most cases as shown in Table 1. The distribution of organisms differed in $\mathrm{HA}$ and CA infections related to increased proportion of enterococci, Pseudomonas species, and Enterobacteriaceae other than Escherichia coli, and a lower proportion due to viridans group streptococci in HA-BSIs as shown in Table 2. Overall, $68(6 \%)$ of episodes of community-onset BSI were of polymicrobial aetiology and this was not different between HA (40/639, 6\%) and CA (28/449, 6\%, $P=0 \cdot 547)$ episodes.

\section{Hospital admission and outcome}

Overall $964(88.6 \%)$ episodes resulted in hospital admission. The acute-care hospital length of stay was a median of 8 (IQR 4-15) days; the total days of acute hospitalization associated with communityonset BSI was 13530 days or 1465 days/100 000 population per year. Of the 964 episodes of community-onset BSI associated with hospital admission, $102(10 \cdot 6 \%)$ died in hospital. Although there was no significant difference in the proportion of cases admitted or length of stay, HA-BSI episodes were associated with higher in-hospital mortality $(72 / 569,12 \cdot 7 \%)$ compared to CA-BSI $(30 / 395,7 \cdot 6 \%, P=0 \cdot 014)$.

\section{DISCUSSION}

We observed that the overall incidence of communityonset BSI was $117 \cdot 8 / 100000$ population and the inhospital case-fatality rate was $10 \cdot 6 \%$. There are few previous population-based studies published for comparison [8]. Filice et al investigated BSIs in Charleston County, South Carolina (population 250000 ) during 1974-1976 with an estimated incidence of community-onset cases of 43/100 000 [8, 16]. Uslan and colleagues reported an overall BSI incidence of 189/100 000 population in Olmsted County, USA (population 124277) from 2003 to 2005 [17]. However, this included $19 \%$ nosocomial cases such that the incidence of community-onset BSI was 153/ 100000 . Neither of these two studies reported casefatality rates associated with community-onset BSI. We previously investigated community-onset BSIs in Calgary (2000-2004, population 1 million) and Victoria (1998-2005, population 360000 ) Canada, and found overall incidence rates of 81.6 and $101 \cdot 2 /$ 100000 , respectively $[19,20]$. Notably the study from Calgary excluded all typical bloodstream contaminants explaining at least in part the lower incidence 


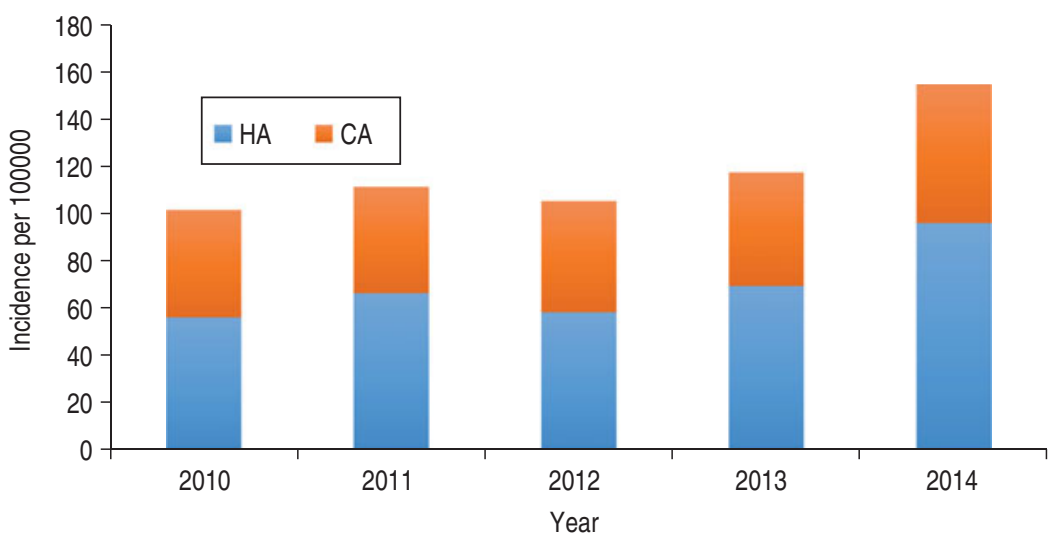

Fig. 1. Annual incidence of community-onset bloodstream infections, Interior Health West, British Columbia, Canada. HA, Healthcare associated; CA, community-associated.

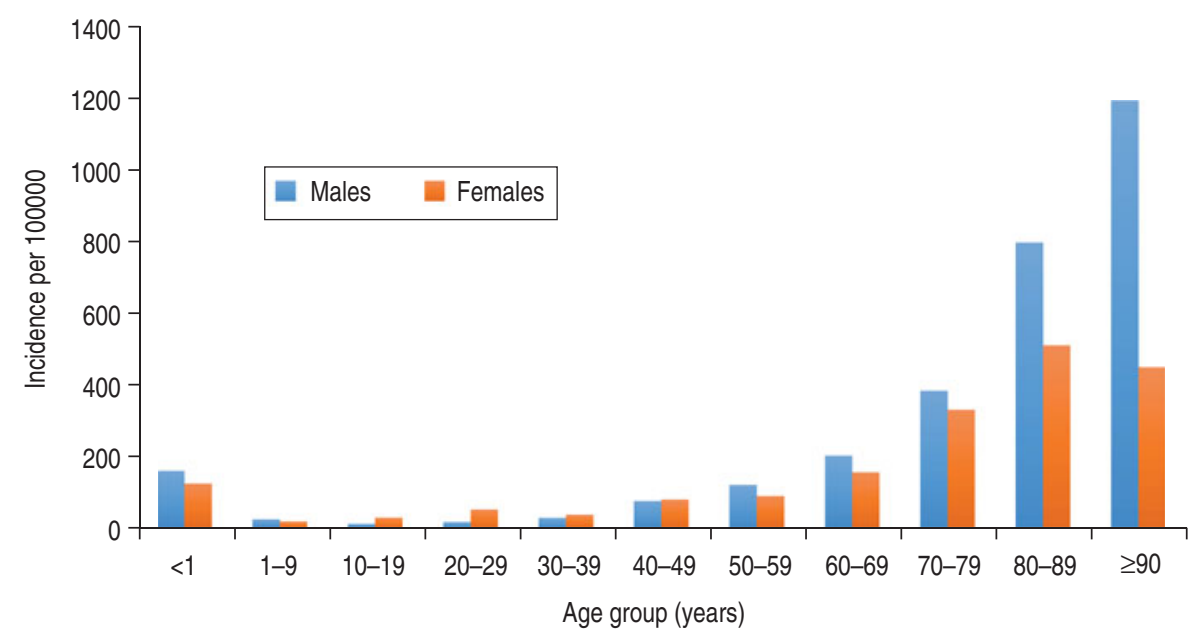

Fig. 2. Age- and gender-specific incidence of community-onset bloodstream infections, Interior Health West, British Columbia, Canada.

Table 1. Clinical focus of community-onset bloodstream infections, Interior Health West, British Columbia, Canada

\begin{tabular}{lllll}
\hline \hline & HA $(\%)$ & CA $(\%)$ & Overall (\%) & $P$ value \\
\hline Primary/no focus & $105(16)$ & $55(12)$ & $160(15)$ & $0 \cdot 0676$ \\
Bone and joint & $39(6)$ & $24(5)$ & $63(6)$ & $0 \cdot 6928$ \\
Soft tissue & $58(9)$ & $32(7)$ & $90(8)$ & $0 \cdot 2658$ \\
Respiratory & $72(11)$ & $59(13)$ & $131(12)$ & $0 \cdot 3457$ \\
Cardiovascular & $29(5)$ & $40(9)$ & $69(6)$ & $0 \cdot 0051$ \\
Intra-abdominal/pelvic & $127(20)$ & $86(19)$ & $213(20)$ & $0 \cdot 8161$ \\
Central nervous system & $1(<1)$ & $11(2)(1)$ & $0 \cdot 0004$ \\
Urinary & $208(33)$ & 449 & $350(32)$ & $0 \cdot 7921$ \\
Total & 639 & 1088 & - \\
\hline \hline
\end{tabular}

HA, Healthcare associated; CA, community associated.

rate in that centre. The in-hospital case-fatality rate in each of these studies was $13 \%$. Sogaard et al. reported an incidence of 112/100 000 for community-onset BSI in Northern Denmark during 1992-2006 with a case- fatality rate of $17 \%$ [18]. While there has been variability observed among regions and time periods, it is evident that community-onset BSI is common and associated with substantial mortality. 
Table 2. Microbiology of community-onset bloodstream infections

\begin{tabular}{lllll}
\hline \hline Isolate & HCA & CA & Overall & $P$ \\
\hline Escherichia coli & $222(35)$ & $180(40)$ & $402(37)$ & $0 \cdot 0744$ \\
Staphylococcus aureus & $108(17)$ & $67(15)$ & $175(16)$ & $0 \cdot 4027$ \\
Methicillin susceptible & 81 & 58 & 139 & $0 \cdot 9267$ \\
Methicillin resistant & 27 & 9 & 36 & $0 \cdot 0570$ \\
Streptococcus pneumoniae & $33(5)$ & $32(7)$ & $65(6)$ & $0 \cdot 1948$ \\
$\beta$-haemolytic streptococci & $47(7)$ & $42(9)$ & $89(8)$ & $0 \cdot 2615$ \\
Enterococcus species & $32(5)$ & $11(2)$ & $43(4)$ & $0 \cdot 0392$ \\
Viridans group streptococci & $14(2)$ & $29(6)$ & $43(4)$ & $0 \cdot 0007$ \\
Other Gram positives & $18(3)$ & $13(3)$ & $31(3)$ & $1 \cdot 0$ \\
Klebsiella species & $44(7)$ & $15(3)$ & $59(5)$ & $0 \cdot 0138$ \\
Other Enterobacteriaceae & $45(7)$ & $12(3)$ & $57(5)$ & $0 \cdot 0013$ \\
Pseudomonas species & $20(3)$ & $5(1)$ & $25(2)$ & $0 \cdot 0380$ \\
Other Gram negatives & $19(3)$ & $16(4)$ & $35(3)$ & $0 \cdot 6041$ \\
Anaerobes & $31(5)$ & $26(6)$ & $57(5)$ & $0 \cdot 4933$ \\
Other & $6(1)$ & $1(<1)$ & $7(1)$ & $0 \cdot 2502$ \\
Total & 639 & 449 & 1,088 & \\
\hline \hline
\end{tabular}

HA, Healthcare-associated; CA, community associated.

We observed that HA community-onset BSIs were more common, were associated with a different distribution of clinical foci and microbiology, and were associated with higher in-hospital mortality than CA infections. In contrast, in their study from Denmark, Sogaard et al. [18] found that CA infections (79/100000) were far more frequent than HA infections (34/100 000). However, their definition for HA was limited to exposure to hospitals (either recent admission or for specialized care). It is likely that a portion of their patients reported with CA would have been classified as HA using the strict definitions proposed by Friedman et al. that we used [3]. Similar to our study, they observed that HA infections were associated with a higher mortality than CA community-onset BSIs. The observations of a higher proportion of methicillinresistant $S$. aureus and mortality associated with HA vs. CA BSIs is consistent with the original study by Friedman et al. [3] and supported by a number of other non-population-based studies conducted in other regions and patient groups [27-31].

While there is a large body of hospital-based literature describing the occurrence and clinical and microbiology of BSIs, population-based studies, especially those investigating a full range of aetiologies concomitantly are uncommon. Population-based studies, by virtue of the fact that all cases of disease occurring in residents of a defined geographical area during a set time period are ascertained, minimize a number of important biases [5, 8, 32, 33]. In our study we used a rigorous methodology. Our study laboratory system performs all blood culture testing for patients in both the outpatient and hospital settings in our region, thus the risk of missing cases or 'ascertainment bias' was minimized. We did not sample from a range of cases but rather included all cases in our population thus minimizing or avoiding the effect of sampling bias. In addition, we utilized strict and explicit definitions for excluding repeat isolates and for determining the presence of and classifying onset of a BSI. Finally, our surveillance region is relatively geographically isolated, our population demographic well defined, and residents were identified by postal code. This allowed us to accurately define the population at risk and exclude extraneous cases (i.e. nonresidents) to minimize the known risk for 'referral bias' $[4,5]$.

While we employed a rigorous study methodology there are some limitations that should be noted. Diagnosis of a BSI requires a positive blood culture. However, if a blood sample is not drawn and sent for culture then by definition a BSI cannot be diagnosed. If this is systematically the case then observed rates will underestimate the true incidence in a population. In our publicly funded system blood cultures are readily available and offered free of charge to patients such that there is no evident barrier to performing this test. While this is not likely a major bias, the possibility does remains that some cases were missed in our population as a result of failure to send cultures. However, this is a limitation of all BSI studies. A second important potential limitation 
of our study is that we could have missed incident cases if residents of our region presented for medical attention outside our region, such as when travelling abroad. While we have no empirical data to suggest the potential magnitude of this bias, we suspect that it is likely a relatively small number. A third limitation of our study is that we conducted the clinical review of cases retrospectively and were limited to data available in the existing medical record. Finally, we only reported crude in-hospital mortality rates as a measure of burden of illness associated with communityonset BSI. Establishment of attributable mortality would have required further multivariable analysis with detailed clinical information such as co-morbid illnesses that were not available in this study [34].

In this study we demonstrate that community-onset BSIs are associated with a significant burden of illness. In addition we document that HA and CA are different as regards occurrence, clinical focus, and microbiology as well as outcome. This study is an important addition to the small body of existing literature investigating the epidemiology of community-onset BSIs at the population level and further documents the need for enhanced efforts to reduce the major adverse effects of these important infections.

\section{DECLARATION OF INTEREST}

None.

\section{REFERENCES}

1. Morin CA, Hadler JL. Population-based incidence and characteristics of community-onset Staphylococcus aureus infections with bacteremia in 4 metropolitan Connecticut areas, 1998. Journal of Infectious Diseases 2001; 184: 1029-1034.

2. Nielsen SL, et al. The daily risk of bacteremia during hospitalization and associated 30-day mortality evaluated in relation to the traditional classification of bacteremia. American Journal of Infection Control 2016; 44: 167-172.

3. Friedman ND, et al. Health care-associated bloodstream infections in adults: a reason to change the accepted definition of community-acquired infections. Annals of Internal Medicine 2002; 137: 791-797.

4. Laupland KB. Population-based epidemiology of intensive care: critical importance of ascertainment of residency status. Critical Care 2004; 8: R431-436.

5. Laupland KB. Defining the epidemiology of bloodstream infections: the 'gold standard' of populationbased assessment. Epidemiology and Infection 2013; 141: 2149-2157.
6. Diekema DJ, et al. Epidemiology and outcome of nosocomial and community-onset bloodstream infection. Journal of Clinical Microbiology 2003; 41: 36553660 .

7. Steckelberg JM, et al. Influence of referral bias on the apparent clinical spectrum of infective endocarditis. American Journal of Medicine 1990; 88: 582-588.

8. Laupland KB, Church DL. Population-based epidemiology and microbiology of community-onset bloodstream infections. Clinical Microbiology Reviews 2014; 27: 647-664.

9. Akoua-Koffi C, et al. Epidemiology of community-onset bloodstream infections in Bouake, central Cote d'Ivoire. New Microbes and New Infections 2015; 7: 100-104.

10. Al-Hasan MN, Eckel-Passow JE, Baddour LM. Impact of healthcare-associated acquisition on communityonset Gram-negative bloodstream infection: a population-based study: healthcare-associated Gram-negative BSI. European Journal of Clinical Microbiology and Infectious Disease 2012; 31: 1163-1171.

11. Anderson DJ, et al. Bloodstream infections in community hospitals in the 21st century: a multicenter cohort study. PLOS ONE 2014; 9: e91713.

12. Hernandez $\mathbf{C}$, et al. Community-onset bacteraemia of unknown origin: clinical characteristics, epidemiology and outcome. European Journal of Clinical Microbiology and Infectious Disease 2014; 33: 1973-1980.

13. Kollef MH, et al. Epidemiology, microbiology and outcomes of healthcare-associated and communityacquired bacteremia: a multicenter cohort study. Journal of Infection 2011; 62: 130-135.

14. Lim CJ, et al. Community-onset bloodstream infection with multidrug-resistant organisms: a matched casecontrol study. BMC Infectious Diseases 2014; 14: 126.

15. Horner $\mathbf{C}$, et al. Escherichia coli bacteraemia: 2 years of prospective regional surveillance (2010-12). Journal of Antimicrobial Chemotherapy 2014; 69: 91-100.

16. Filice GA, et al. Bacteremia in Charleston County, South Carolina. American Journal of Epidemiology 1986; 123: 128-136.

17. Uslan DZ, et al. Age- and sex-associated trends in bloodstream infection: a population-based study in Olmsted County, Minnesota. Archives of Internal Medicine 2007; 167: 834-839.

18. Sogaard M, et al. Temporal changes in the incidence and 30-day mortality associated with bacteremia in hospitalized patients from 1992 through 2006: a population-based cohort study. Clinical Infectious Diseases 2011; 52: 61-69.

19. Laupland KB, et al. Burden of community-onset bloodstream infection: a population-based assessment. Epidemiology and Infection 2007; 135: 1037-1042.

20. Laupland KB, et al. Population-based laboratory assessment of the burden of community-onset bloodstream infection in Victoria, Canada. Epidemiology and Infection 2013; 141: 174-180.

21. Anon. Interactive Health Geographies, BC Statistics (http://bcstats.gov.bc.ca/StatisticsBySubject/Geography/ ReferenceMaps/Health.aspx). Accessed November 18, 2015. 
22. Anon. Department of Medicine, Royal Inland Hospital (http://www.kamloopsmedicine.com/hospital). Accessed 18 November 2015.

23. Leal J, et al. Development of a novel electronic surveillance system for monitoring of bloodstream infections. Infection Control and Hospital Epidemiology 2010; 31: 740-747.

24. Laupland KB, Gregson DB, Church DL. Validity of calendar day-based definitions for community-onset bloodstream infections. BMC Research Notes 2015; 8: 123.

25. Anon. Population estimates, BC stats (http://www.bcstats. gov.bc.ca/StatisticsBySubject/Demography/Population Estimates.aspx). Accessed 15 September 2015.

26. Laupland KB, et al. Invasive group A streptococcal disease in children and association with varicella-zoster virus infection. Ontario Group A Streptococcal Study Group. Pediatrics 2000; 105: E60.

27. Shorr AF, et al. Healthcare-associated bloodstream infection: A distinct entity? Insights from a large U.S. database. Critical Care Medicine 2006; 34: 2588-2595.

28. De Bus L, et al. Microbial etiology and antimicrobial resistance in healthcare-associated versus communityacquired and hospital-acquired bloodstream infection in a tertiary care hospital. Diagnostic Microbiology and Infectious Diseases 2013; 77: 341-345.

29. Valles $\mathbf{J}$, et al. Bloodstream infections in adults: importance of healthcare-associated infections. Journal of Infection 2008; 56: 27-34.

30. Wu HS, et al. Characteristics of healthcare-associated and community-acquired Klebsiella pneumoniae bacteremia in Taiwan. Journal of Infection 2012; 64: 162-168.

31. Rodriguez-Bano J, et al. Epidemiology and clinical features of community-acquired, healthcare-associated and nosocomial bloodstream infections in tertiary-care and community hospitals. Clinical Microbiology and Infection 2010; 16: 1408-1413.

32. Rempel O, Pitout JD, Laupland KB. Antimicrobial resistance surveillance systems: Are potential biases taken into account? Canadian Journal of Infectious Diseases and Medical Microbiology 2011; 22: e24-28.

33. Rempel OR, Laupland KB. Surveillance for antimicrobial resistant organisms: potential sources and magnitude of bias. Epidemiology and Infection 2009; 137: 1665-1673.

34. Abernethy JK, et al. Thirty day all-cause mortality in patients with Escherichia coli bacteraemia in England. Clinical Microbiology and Infection 2015; 21: e251-258. 